\title{
A pellet tracking system for hadron physics experiments
}

\author{
A. Pyszniak ${ }^{1,2, a}$, H. Calén ${ }^{2}$, K. Fransson² ${ }^{2}$ M. Jacewicz² ${ }^{2}$ T. Johansson², and Z. Rudy ${ }^{1}$ \\ ${ }^{1}$ Institute of Physics, Jagiellonian University, PL-30-059 Cracow, Poland \\ ${ }^{2}$ Department of Physics and Astronomy, Uppsala University, SE-75120, Uppsala, Sweden
}

\begin{abstract}
Frozen microspheres of hydrogen (pellets) are used as targets in the hadron physics experiment WASA (Forschungszentrum Jülich, Germany) [1] and will also be used in the future PANDA experiment at FAIR (GSI, Darmstadt, Germany) [2]. The interaction region is defined by the overlap of the pellet stream and the accelerator beam and has a size of a few millimeters. One would like to know the interaction point more precisely, to have better possibility to reconstruct particle tracks and events. One would also like to suppress background events that do not originate in a pellet, but e.g. may occur in residual gas in the beam pipe. A solution is provided by the presented pellet tracking system, for which a prototype [3] has been developed in Uppsala. The goal is to track individual pellets in order to know their position at the time of an interaction. The design of such a system, simulation techniques and results are presented.
\end{abstract}

\section{Introduction}

Pellets with a diameter of 25-30 $\mu \mathrm{m}$ are generated 2 - 3 meters above the interaction region, to which they travel with a velocity around $80 \mathrm{~m} / \mathrm{s}$ inside a thin pipe (the space between pellet generation point and the interaction point is occupied by the particle detector system). The distance between the pellets is in the order of a few millimeters and they form a stream of a few millimeters in diameter.

The tracking of pellets will be realized using lasers and fast line-scan (i.e. "one dimensional") CCD cameras. The cameras, placed at different levels along the pellet stream, measure pellets position and time of passage (Figure 1). Then, the information from many cameras will be put together to identify and reconstruct the track of each pellet. Information about the pellet position in the interaction region at the time of an interaction will be used in the analysis of the experimental data. To be useful, the tracking system must be highly efficient and provide tracking information for essentially all pellets that pass through the interaction region.

Tests and measurements at the Uppsala Pellet Test Station (UPTS, Figure 2) are used in the development of the tracking system. At the pellet generator, there are detection possibilities at the droplet chamber (DC), at the exit of the vacuum injection capillary (VIC) and above the turbo pump section (PTR gen). Below the pumps, in the lower part there are detection possibilities just above the skimmer and at two levels in a prototype pellet tracking chamber (see the figure).

ae-mail: andrzej.pyszniak@gmail.com 


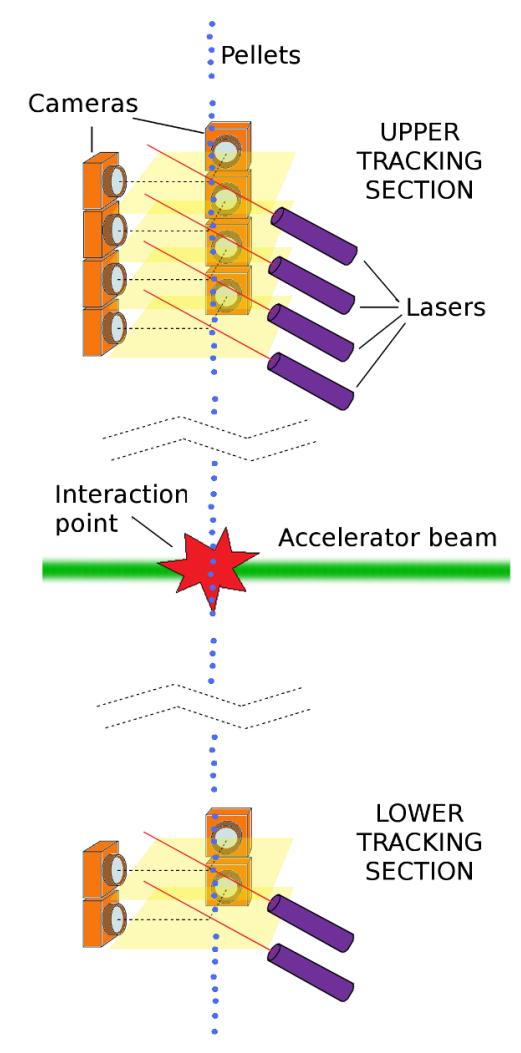

Figure 1: Principle of operation of a pellet tracking system

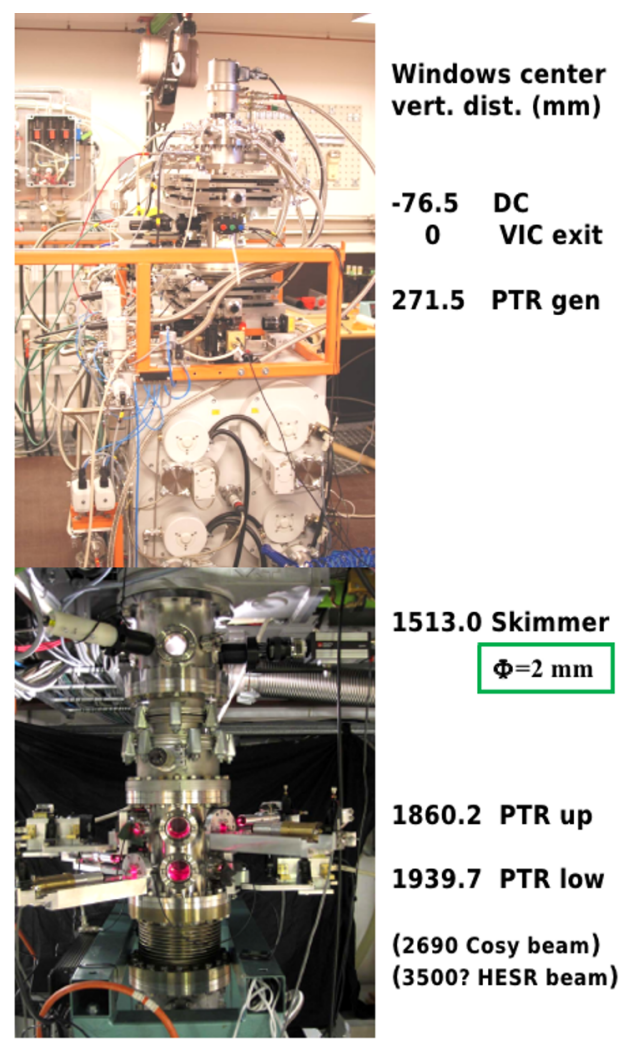

Figure 2: The UPTS setup with the different pellet detection levels indicated

\section{Measurements}

Measurements are done at different distances between measurement levels, with different detection efficiencies and at different pellet rates. The example spectrum in Fig. 3 is obtained by comparing times of pellet signals recorded by the upper and lower camera. The peak seen in the spectrum (correlation peak) is due to signals from the same pellet at the two cameras. The background is a result of signals from different pellets. The position of the correlation peak indicates pellet time of flight between measurement levels and the peak width is a result of velocity spread $(\Delta v / v \approx 0.5 \%)$.

Making a cut on the time difference between between pellet signals at two measurement levels, one can select measurements, that with high probability correspond to the same pellet. The measured pellet positions are then used to extrapolate the pellet track back to the VIC (nominal generation point).

The left plot in Fig. 4 shows the extrapolated transverse position of pellet tracks in the direction of the VIC. By knowing the nominal position of the VIC exit, one can obtain a resolution of the reconstruction of $\sigma \approx 100 \mu \mathrm{m}$. Taking into account the distance to the measurement levels and the distance between them, the detection resolution at the measurement levels is estimated to be $\approx 20 \mu m$. The results of the measurements are well described by the MC simulation (right plot in Fig. 4). 

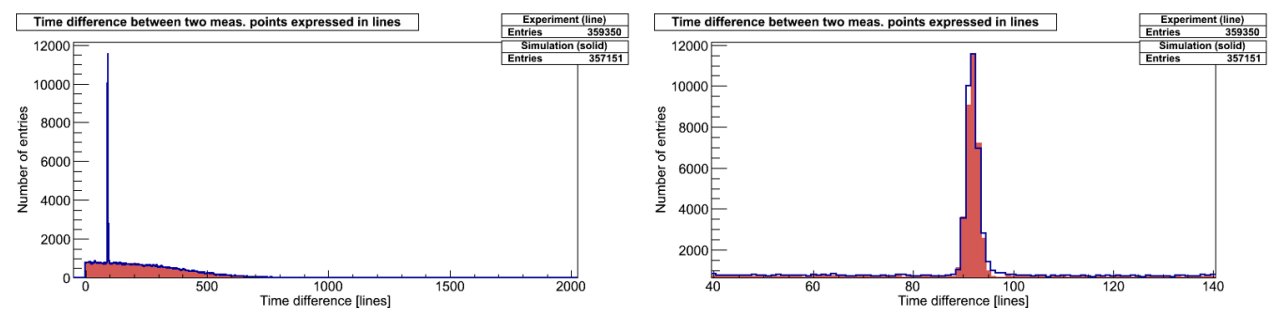

Figure 3: Time difference between pellets recorded at PTR lower and PTR upper (distance $80 \mathrm{~mm}$ ) with magnification of the correlation peak region. Camera period: $12.5 \mu \mathrm{s}$. Experiment - blue line, MC - solid red
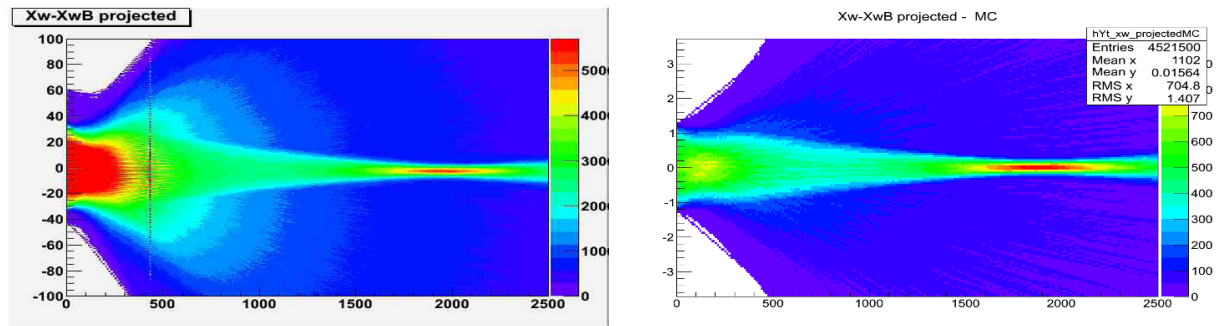

Figure 4: Extrapolated transverse positions of pellet tracks from experimental data taken at UPTS (left plot) and corresponding simulations (right plot). In the left plot the scale on the x-axis is in millimeters and on the $y$-axis in pixels $(\approx 37 \mu \mathrm{m})$. In the right plot the scale is in millimeters.

\section{Design simulations}

The aim of the design simulation studies is to check how the expected position resolution and tracking efficiency for different design variants meets the goals and requirements from the physics experiment and find out how they depend on various parameters describing the pellet and pellet stream properties and the detection process (for details see Ref. [4]). The geometrical conditions and tracking equipment configurations are based on a conceptual design for PANDA where two $400 \mathrm{~mm}$ long sections of the vertical pellet beam pipe are reserved for the tracking system, one at the pellet generator and the other one at the dump. At each section four measurement levels can be accommodated.

In the design simulations we calculate the true position and time of each pellet at the measurement levels. This information is then used to simulate the measurements of pellet position and time. The simulation takes into account various aspects of the measurement, as illumination, optical imperfections and electronics noise. Parameters in the simulations are the pellet physical size, brightness, different distortions due to optical effects, camera pixels size and structure of the camera cycle (period and exposure time). Realistic parameter distributions are taken from UPTS experiments.

During the pellet tracking procedure we take each pellet recorded at the first measurement level as a start and for this pellet we want to collect all information needed to reconstruct the time and position of this pellet at the interaction region. To reconstruct the track of the pellet we need to know the position of the pellet at the following measurement points. To select the correct measurement from among all others, we use the mean velocity of the pellet stream and distance to the second measurement level, to get the expected arrival time. We look for measurements around this time, in a certain time window, and the measurement closest to the expected time is assumed to be the measurement of the pellet in the track. Its time is used to calculate a new, improved velocity, which is then used to search for the 
pellet at the next measurement levels. This is continued until the last measurement level. The whole procedure is repeated for all pellets recorded at the first measurement level. Having information from all measurement levels, one fits the pellet tracks. The pellet start position (VIC exit) may also be used as an additional point in the track fitting.

One then obtains the tracking resolution in the simulations, by comparing reconstructed and true pellet position at the interaction region. Example resolution plots are shown in Fig. 5.
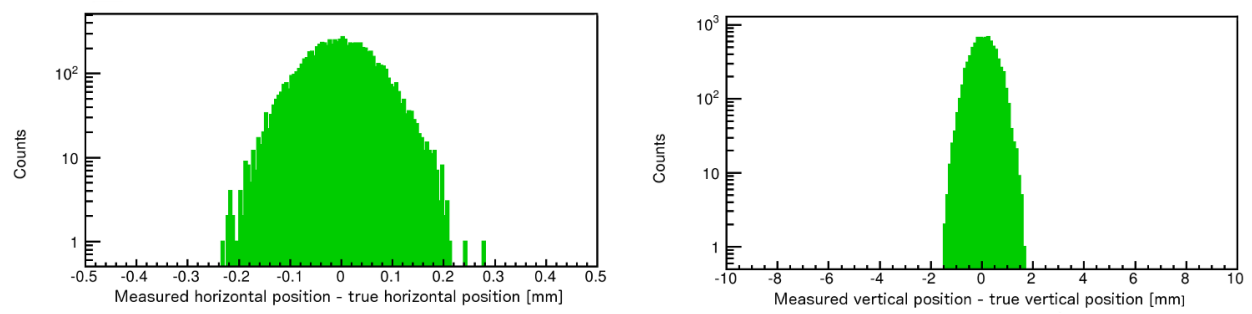

Figure 5: Examples of pellet position resolution from tracking. The left plot is for one of the horizontal coordinates and the right plot is for the vertical coordinate.

The resolution in the horizontal directions depends directly on the position measurement resolution and on the distance between the measurement levels. A resolution of $\sigma \approx 250 \mu m$ is obtained when using information from the upper tracking section only. The resolution is good enough for an accurate consistency check aimed at verifying whether a track could originate from the VIC exit (situated about $700 \mathrm{~mm}$ above the tracking section). If this is the case the pellet stream position at the VIC can be used as an additional point on the track fit, substantially increasing the lever arm. Then a position resolution of $\sigma \approx 70 \mu \mathrm{m}$ is obtained. For the reference conditions, the resolution in the vertical direction depends mainly on the accuracy in the velocity determination i.e. on the time resolution from the cameras and the distance between first and last level in the measurement section. The additional measurement levels in between, improve the resolution slightly $(\approx$ $30 \%$ ) and results in a resolution of $\sigma \approx 0.8 \mathrm{~mm}$. The lower tracking section at the dump might improve the resolution and can also be used for tuning and monitoring of the tracking performance.

The tracking system should provide useful i.e. correct tracking information for as many hadronic events as possible. For a certain number of pellets present in the interaction region, the efficiency is defined as the probability that the number of pellets found by the tracking is the right number. From the results of these studies one can deduce that for the reference conditions about $70 \%$ of the hadronic events (i.e. when pellets are in the beam region) would have correct information from the tracking system and for about $50 \%$ of the events there would be unambiguous position information.

\section{Acknowledgements}

The project is supported by Forschungszentrum Jülich COSY-FFE, European Commission Framework Program 7, Swedish Research Council, Foundation for Polish Science (MPD program) and Polish National Science Center.

\section{References}

[1] WASA-at-COSY Collaboration: H.-H. Adam et al., arXiv:nucl-ex/0411038 (2004)

[2] W. Erni et al., PANDA Collaboration, http://arxiv.org/abs/0903.3905v1 (2009)

[3] H. Calén et al., Forschungszentrum Jülich IKP Annual Report, (2011)

[4] A. Pyszniak et al., Simulation studies for design of pellet tracking systems, Project report, http://www.physics.uu.se/en/np/panda/pub/ (2013) 